\title{
Neuromyelitis optica: a pilot study of clinical presentation and status of serological biomarker AQP4 among patients admitted to a tertiary centre in NCNS, Sudan
}

\author{
Etedal Ahmed AbuElbasher Ibrahim ${ }^{1,2^{*}} \mathbb{1}$, Fatima Gammer ${ }^{2}$ and Alsadig Gassoum²,3
}

\begin{abstract}
Background: Neuromyelitis optica (NMO) is a demyelinating disease primarily affecting the optic nerves and spinal cord. It is distinguished from other demyelinating conditions by the presence of AQP4-lgG and serum aquaporin 4 (AQP4), found mainly in the blood-brain barrier. This descriptive study was conducted from January 2015 to June 2018 at the National Center for Neurological Sciences (NCNS) in Khartoum, Sudan. All participants were Sudanese patients diagnosed with NMO. In our study the selection of cases was based on Dean Wingerchuk diagnostic criteria (2006), which states that the diagnosis of NMO should meet two absolute criteria and two supportive criteria. The absolute criteria are myelitis and optic neuritis, whereas supportive criteria include radiological findings obtained from brain and spinal cord MRI. Furthermore, AQP4-IgG levels were measured from cerebrospinal fluid (CSF) and serum using immunofluorescence. Data were collected by a pre-designed questionnaire and analyzed using SPSS version 17. A p value $<0.05$ was considered statistically significant.

Results: A total of 31 patients were enrolled in this study [6 male (19.4\%) and 25 female (80.6\%)]. The mean age was $38 \pm 12.8$ years. Motor and visual difficulties were the initial symptoms and occurred in 21 (67.7\%) and 10 (32.3\%) patients, respectively. Fundoscopy confirmed optic atrophy in 22 (71.0\%) patients. The course of the disease revealed one relapse in 21 patients (67.7\%). Seropositive AQP4-IgG were seen in 23 patients (79.31\%). There was a significant correlation between AQP4 and response to treatment $(p \leq 0.038)$. The correlation between serum AQP4-IgG, showed that, complete improvement was detected in 2 patients (6.9\%) one of them was positive and the other was negative, 20 (69.0\%) patients presented with some disability, among them 18 (62.1\%) were positive and 2 (6.9\%) were negative, while 7 patients showed no improvement (24.1\%) 4 out of them were positive (13.8\%) and 3 were negative (10.3\%).
\end{abstract}

Conclusion: At the initial presentation of $\mathrm{NMO}$, longitudinal myelitis was observed more frequently than optic neuritis. More than two third of the patients showed strong seropositivity for serum AQP4. Most seropositive patients showed a good response to treatment but with residual disabilities.

Keywords: AQP4-IgG, Neuromyelitis optica, Serological biomarkers, Sudan

*Correspondence: eetedalibrahim@yahoo.com

${ }^{1}$ Alneelain University, Faculty of Medicine, Khartoum, Sudan

Full list of author information is available at the end of the article

\section{Background}

Neuromyelitis optica (NMO) is a demyelinating disease primarily affecting the optic nerves and spinal cord [1]. It is characterized by the presence of optic neuritis and/ or myelitis. It can be monophasic but $73 \%$ to $90 \%$ of cases have a relapsing course [2]. 
The disease is distinguished from other demyelinating conditions by the presence of AQP4-IgG which is expressed by the gene AQP4, and is mainly found in the blood-brain barrier of the central nervous system [3]. The gene belongs to the AQP4 family whose members conduct water through cell membranes [3]. Their main function is to transport water and maintain homeostasis within the nervous system. AQP4 is a predominant target in neuromyelitis optica. Detection of this protein in the serum of the patient reflects the disease activity. The diagnosis of the disease can be made by the associated clinical characteristics, MRI findings of a longitudinal lesion involving more than 3 consecutive vertebrae, and the presence of AQP4-IgG in the CSF as well as positive serum AQP4 [4-10].

\section{Objective}

To study the clinical presentation and serological biomarker status and disease outcome and its correlation with AQP4 among Sudanese patients at the National Center of Neurological Sciences (NCNS) in Khartoum, Sudan, from January 2015 to June 2018.

\section{Methods}

This was a descriptive study. The study included $31 \mathrm{NMO}$ patients with diagnosis based on the following: clinical presentation with optic neuritis and myelitis confirmed by MRI of the brain with FLAIR and MRI of cervical and dorsalspine, as well as positivity of serum Aquaporin 4 and CSF for AQP4-IgG (performed using ELISA RSR Ltd, Cardiff, UK). Other investigations were done to exclude mimickers such as: serum and CSF oligoclonal bands to rule out multiple sclerosis (MS), complete blood count, erythrocyte sedimentation rate (ESR), C-reactive protein, anti-nuclear antibody (ANA), anti- double-stranded DNA antibodies (Anti-dsDNA), and ACLP antibodies to exclude vasculitis. The blood and CSF samples were analyzed by immunofluorescence.

In our study the selection of cases was based on Dean Wingerchuk diagnostic criteria (2006), which require that patients meet both the two absolute criteria and any two of the supportive criteria [11].

\section{Absolute criteria}

1. Optic neuritis.

2. Acute myelitis.

\section{Supportive criteria}

1. MRI of the brain not meeting the criteria for MS at disease onset.
2. MRI of the spinal cord with contiguous T2-weighted signal abnormality extending over three or more vertebral segments, indicating a relatively large lesion in the spinal cord.

3. AQP4-IgG seropositive status (The AQP4-IgG test checks the existence of antibodies against the Aquaporin 4 antigen).

Data was collected by a comprehensive structured questionnaire. It covered demographics and medical data in addition to descriptive statistics in terms of frequency, and tables with percentages for the qualitative data. Data was collected, reviewed and analyzed using SPSS version 17. Furthermore; relations were analyzed using crosstabulation and Chi square test. A $p$ value of $<0.05$ was considered statistically significant.

The study was ethically approved by the National Center for Neurological Sciences ethical committee, Khartoum, Sudan. Written consent was taken from all the patients who participated in the study.

\section{Results}

\section{Demographic characteristics}

A total of 31 Sudanese patients with NMO were included in this study. Six (19.4\%) of the patients were males and 25 (80.6\%) were females. Seventeen (54.8\%) patients were more than 40 years and the mean age was 38.74 \pm 12.85 years. In this study the distribution of $\mathrm{NMO}$ patients indicated 18 (58.1) patients from the north of Sudan, the majority of the tribes which reside in this area were belonging to Afro-asiatic group, while 8 (25.8) from eastern Sudan, which were belonging also to Afro-asiatic group, and 5 patients (16.1\%) from the western Sudan, the most common tribes in this state are from Nilo-Saharan group. From our data registry we found that, the total number of NMO patients during the last year was 38 , and accordingly the incidence will be 0.81 .

Motor symptoms were reported first in 21 patients (67.7\%) and visual symptoms in 10 patients (32.3\%). Among the 10 patients with visual symptoms, 8 of them $(80.0 \%)$ had complete visual loss and more frequent in bilateral optic neuritis, the frequency of bilateral optic neuritis was $41.9 \%$ and unilateral optic neuritis represented 19.4\%, 1 (10\%) had impaired visual acuity, and 1 $(10 \%)$ had impaired acuity as well as color vision. Among the 21 patients with motor symptoms, 19 (90.5\%) had lower limb weakness, 1 (4.76\%) had upper limb weakness, and 1 (4.76\%) patient had both. Seventeen (54.8\%) patients had sensory symptoms while 12 (38.7\%) had sphincteric disturbances. Table 1 describes these findings.

The interval between visual and motor symptoms varied between days, weeks, months, and years in 5 
Table 1 Demographic characteristics of the study participants

\begin{tabular}{|c|c|c|c|}
\hline Variables & Findings & No & Percentage (\%) \\
\hline \multirow[t]{2}{*}{ Gender } & Male & 6 & 19.4 \\
\hline & Female & 25 & 80 \\
\hline \multirow[t]{6}{*}{ Age in years } & $16-20$ & 4 & 12.9 \\
\hline & $21-25$ & 3 & 9.7 \\
\hline & $26-30$ & 3 & 9.7 \\
\hline & $31-35$ & 3 & 9.7 \\
\hline & $36-40$ & 1 & 3.2 \\
\hline & $>40$ & 17 & 54.8 \\
\hline \multirow[t]{2}{*}{ Symptoms } & Visual & 10 & 32.3 \\
\hline & Motor & 21 & 67.7 \\
\hline \multirow[t]{3}{*}{ Visual status } & Impairment of visual acuity & 1 & 10 \\
\hline & Complete visual loss & 8 & 80 \\
\hline & Impairment of visual acuity \& color vision & 1 & 10 \\
\hline \multirow[t]{4}{*}{ Disease duration since the onset } & Days & 5 & 16.1 \\
\hline & Weeks & 6 & 19.4 \\
\hline & Months & 10 & 32.3 \\
\hline & Years & 10 & 32.3 \\
\hline \multirow[t]{3}{*}{ Visual acuity } & Blindness & 13 & 41.9 \\
\hline & Normal & 12 & 38.7 \\
\hline & Impaired & 6 & 19.4 \\
\hline \multirow[t]{2}{*}{ Fundus examination } & Optic atrophy & 22 & 71.0 \\
\hline & Normal & 9 & 29.0 \\
\hline \multirow[t]{5}{*}{ Motor system findings } & Paraparises & 6 & 19.4 \\
\hline & Paraplegia & 16 & 51.6 \\
\hline & Quadriparesis & 4 & 12.9 \\
\hline & Quadriplegia & 5 & 16.1 \\
\hline & Complete improvement & 6 & 19.4 \\
\hline \multirow[t]{2}{*}{ Clinical course } & Single relapse & 21 & 68 \\
\hline & Relapsing & 10 & 32 \\
\hline \multirow[t]{2}{*}{ MRI brain } & Normal & 23 & 74 \\
\hline & Abnormal & 8 & 26 \\
\hline \multirow[t]{2}{*}{ MRI spinal cord } & Normal & 2 & 6 \\
\hline & Abnormal & 29 & 94 \\
\hline \multirow[t]{2}{*}{ CSF NMO-IgG } & $+V e$ & 25 & 79 \\
\hline & $-V e$ & 6 & 21 \\
\hline \multirow[t]{2}{*}{ Serum aquaporin-4 antibody } & $+V e$ & 25 & 79 \\
\hline & $-V e$ & 6 & 21 \\
\hline \multirow[t]{2}{*}{ Treatment } & IV methylprednisolone followed by oral & 5 & 16 \\
\hline & IV methylprednisolone followed by oral+ azathioprine & 26 & 84 \\
\hline \multirow[t]{3}{*}{ Outcomes } & Complete improvement & 2 & 6 \\
\hline & Improvement with disability & 22 & 71 \\
\hline & No improvement & 7 & 23 \\
\hline
\end{tabular}

(16.1\%), 6 (19.4\%), 10 (32.3\%), and 10 (32.3\%) patients, respectively. Twenty-one patients $(67.7 \%)$ had one attack whereas 10 (32\%) had a history of many attacks (Table 1).

Interestingly, one patient (3.2\%) had a family history of NMO. All patients (100\%) had normal higher-order functions. Among the visual symptoms, 13 (41.9\%) patients had blindness and $6(19.4 \%)$ patients had impaired visual acuity while $12(38.7 \%)$ had normal visual acuity. Seventeen patients (54.8\%) had normal color vision in contrast to $14(45.2 \%)$ patients who had 
abnormal color vision. In addition, $17(54.8 \%)$ of the patients had central scotoma. Fundoscopy showed that $22(71.0 \%)$ of the patients had optic atrophy (Fig. 1) while the rest had normal optic discs. Other cranial nerves were normal in 28 patients (90.3\%) but were found to be abnormal in 3 (9.7\%) (Table 1$)$.

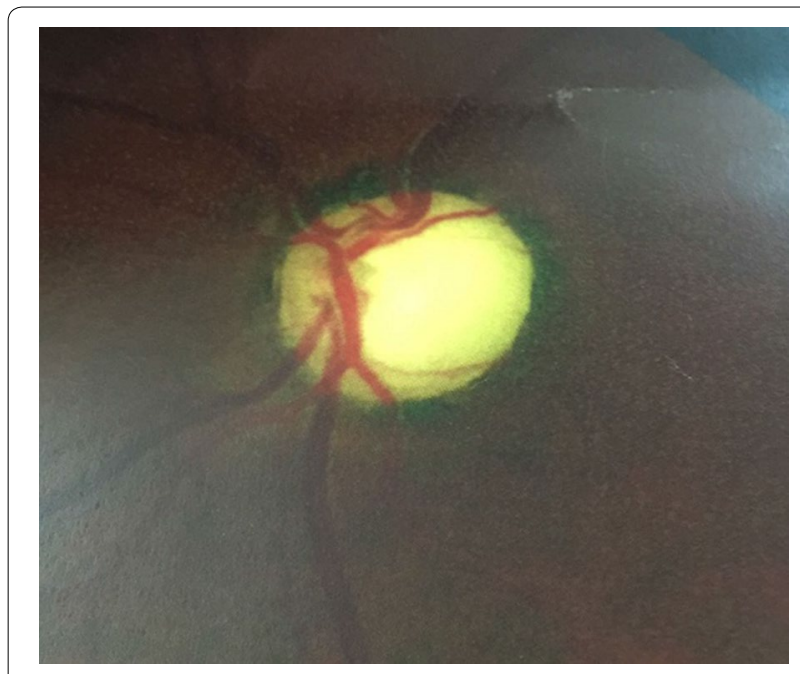

Fig. 1 Optic atrophy in a patient with NMO presented with reduce vision both eyes and lower limbs weakness, over 2 month duration
Motor system impairments ranged from paraparesis which was observed in $6(19.4 \%)$ patients to paraplegia in 16 patients (51.6\%), quadriparesis in 4 (12.9\%) patients. and quadriplegia in 5 patients (16.1\%). Among patients with a relapsing course, 5 patients $(50.0 \%)$ had 2 attacks, 4 patients (40\%) had three attacks, and 1 patient $(10 \%)$ had more than three attacks. We did not find any previous registered data regarding the prevalence of NMO in Sudan. Simultaneously, we studied 65 patients with demyelinating disease (multiple sclerosis) in Sudan (2018) [12]. According to disability score, 14 patients EDSS score 8, 9 patients score 9, 5 patients score 7.5, 1 patient score 5,1 patient score 4 and 1 patient score 3 .

MRI of the brain was normal in 23 patients (74.2\%); however it was abnormal in 8 patients $(25.8 \%)$ as exemplified in Fig. 2. Similarly, MRI of the cervical spine was normal in 2 patients (6.5\%) but was abnormal in 29 patients (93.5\%); it showed longitudinal hyper-intense lesion involving more than 3 consecutive vertebrae (Table 1).

1. In this study when CSF analysis was performed in 31 patients it was positive for AQP4-IgG in 23 patients (79.31\%) and negative in 6 patients (20.69\%). The results of serum Aquaporin4 were positive in 23 patients $(79.31 \%)$ and negative in 6 patients
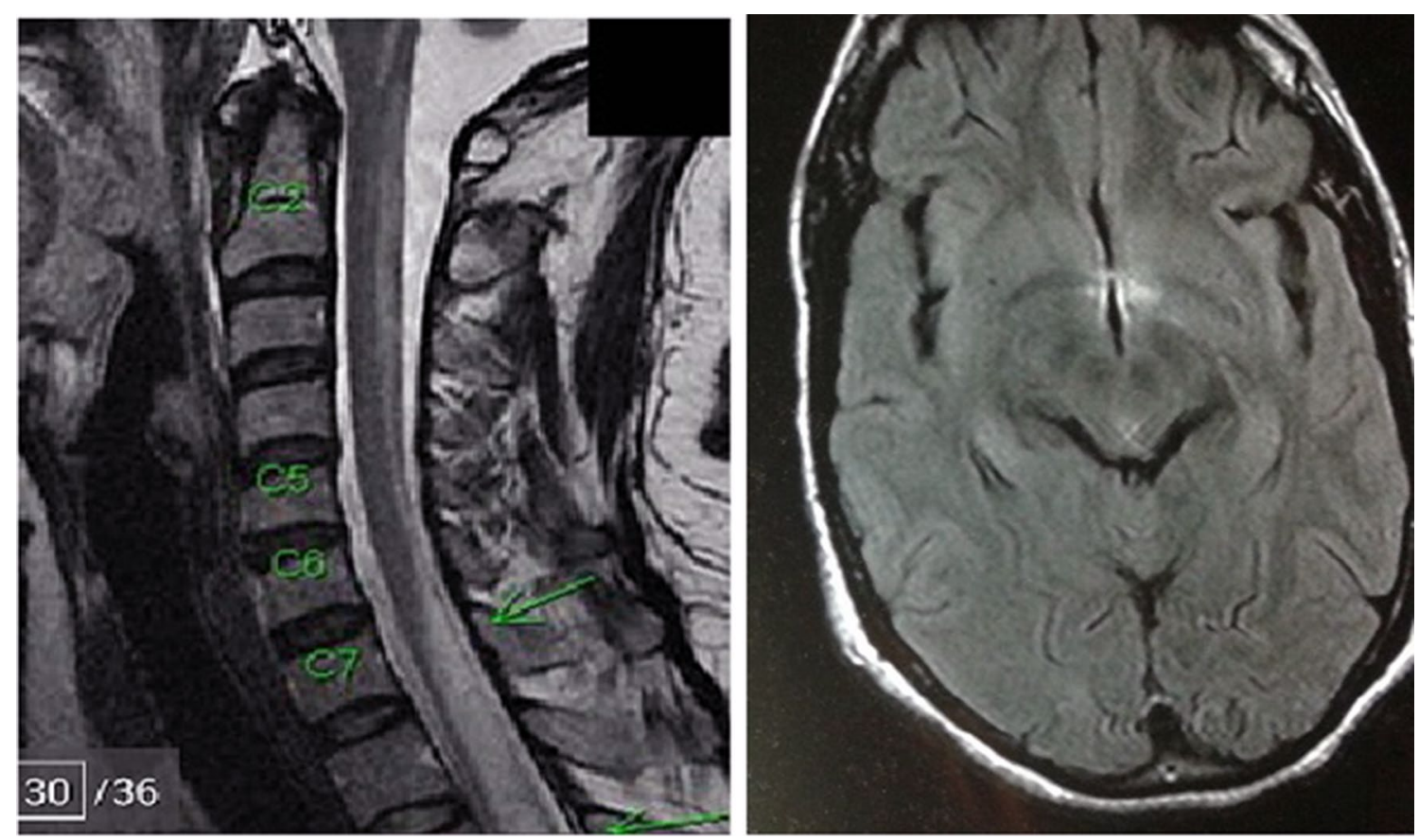

Fig. $2 \mathrm{MRI}$ of the brain showing hyper-intensity in the aquaporin4 area, and MRI of the cervical spine showing longitudinal hyper-intensities in more than 3 consecutive vertebrae in a patient with NMO presented with reduce vision both eyes and lower limbs weakness, over 2 month duration 
(20.29\%), those 6 patients in the study with negative CSF AQP4-IgG were also negative for serum AQP4IgG. Oligoclonal bands were negative in 29 patients (93.5\%). The correlation between serum AQP4-IgG, showed that, complete improvement was detected in 2 patients $(6.9 \%)$ one of them was positive and the other was negative $20(69.0 \%)$ patients presented with some disability, among them 18 (62.1\%) were positive and $2(6.9 \%)$ were negative, while 7 patients showed no improvement (24.1\%) 4 out of them were positive (13.8\%) and 3 were negative (10.3\%).

A majority of the studied population (26, 83.9\%) received treatment in the form of intravenous methylprednisolone $1 \mathrm{gm} /$ day for 5 days followed by oral prednisolone $60 \mathrm{mg} /$ day tapered down according to the response combined with azathioprine $150 \mathrm{mg} /$ day while 5 patients $(16.1 \%)$ did not receive azathioprine.

Among the patients, 22 (71.0\%) showed improvement with some remaining disability, whereas 2 patients (5.6\%) showed complete recovery and 7 (22.6\%) demonstrated no improvement (Table 1). The results of correlation

Table 2 Correlation between outcome and serum aquaporin-4 antibody among the study population

\begin{tabular}{|c|c|c|c|c|}
\hline \multirow[t]{2}{*}{ Outcome } & & \multicolumn{2}{|c|}{$\begin{array}{l}\text { Serum } \\
\text { aquaporin-4 } \\
\text { antibody }\end{array}$} & \multirow[t]{2}{*}{ Total } \\
\hline & & +ve & -ve & \\
\hline \multirow[t]{2}{*}{ Complete improvement } & Count & 1 & 1 & 2 \\
\hline & $\%$ of total & $3.4 \%$ & $3.4 \%$ & $6.9 \%$ \\
\hline \multirow[t]{2}{*}{ Improvement with some disability } & Count & 18 & 2 & 20 \\
\hline & $\%$ of total & $62.1 \%$ & $6.9 \%$ & $69.0 \%$ \\
\hline \multirow[t]{2}{*}{ No improvement } & Count & 4 & 3 & 7 \\
\hline & $\%$ of total & $13.8 \%$ & $10.3 \%$ & $24.1 \%$ \\
\hline \multirow[t]{2}{*}{ Total } & Count & 23 & 6 & 29 \\
\hline & $\%$ of total & $79.3 \%$ & $20.7 \%$ & $100.0 \%$ \\
\hline
\end{tabular}

P-value $=0.104$ between the outcome and serum AQP4are displayed in Table 2, and those of the correlation between treatment and serum AQP4 are displayed in Table 3.

Figure 1 shows optic atrophy in a 37-year-old Sudanese female with NMO.

Figure 2 shows MRI of the brain with hyper-intensity in the area of AQP4, and MRI of the cervical spine with longitudinal hyper-intensity involving more than 3 consecutive vertebrae.

\section{Discussion}

Our study is the first comprehensive attempt to estimate the frequency of NMO and AQP4-IgG seropositivity in Sudanese patients. NMO seemed to be a rare disease in Sudan but our study suggests that it is probably common. In this study, females were more often affected $(80.6 \%$ of the study population). This finding is similar to those of previous studies from Caucasians, French West Indies, Austria and Cuba that estimated female predominance in $66-88 \%$ of the patients [13-16].

Our study revealed that 17 patients $(54.8 \%)$ were above the age of 40 years. A study from Cuba described a mean age of 35 years [17] whereas other studies reported different mean age of patients $[13,15,18]$.

The distribution of NMO patients in this study indicated, 18 (58.1) patients came from the north of Sudan, and the majority of the tribes which reside in this area were belonging to Afro-asiatic group, while 8 (25.8) from eastern Sudan, which were belonging also to Afro-asiatic group, and 5 patients (16.1\%) from the western Sudan, the most common tribes in this state are from Nilo-Saharan group.

In our study, motor symptoms secondary to myelitis were observed more often than visual symptoms due to optic neuritis (67.7\% vs. $32.2 \%$ respectively). Among the 10 patients with visual symptoms, 8 of them $(80.0 \%)$ had complete visual loss and more frequent in bilateral optic neuritis, the frequency of bilateral optic neuritis was $41.9 \%$ and unilateral optic neuritis represented $19.4 \%$,

Table 3 Correlation between treatment and serum aquaporin-4 antibody among the study population

\begin{tabular}{|c|c|c|c|c|}
\hline \multirow[t]{2}{*}{ Treatment } & & \multicolumn{2}{|c|}{ Serum aquaporin- 4 antibody } & \multirow[t]{2}{*}{ Total } \\
\hline & & +ve & - ve & \\
\hline \multirow[t]{2}{*}{ IV methylprednisolone followed by oral prednisolone } & Count & 1 & 2 & 3 \\
\hline & $\%$ of total & $3.4 \%$ & $6.9 \%$ & $10.3 \%$ \\
\hline \multirow{2}{*}{$\begin{array}{l}\text { IV methylprednisolone followed by oral prednisolone + azathio- } \\
\text { prine }\end{array}$} & Count & 22 & 4 & 26 \\
\hline & $\%$ of total & $75.9 \%$ & $13.8 \%$ & $89.7 \%$ \\
\hline \multirow[t]{2}{*}{ Total } & Count & 23 & 6 & 29 \\
\hline & $\%$ OfTotal & $79.3 \%$ & $20.7 \%$ & $100.0 \%$ \\
\hline
\end{tabular}

P-value $=0.038$ 
1 (10\%) had impaired visual acuity, and 1 (10\%) had impaired acuity.

This is similar to the findings from previous studies in which the presenting symptoms were usually either secondary to optic neuritis in $35 \%$ of the patients or due to transverse myelitis in 70\% [19-21].

Complete visual lost during the attack is very high compared to other cohort studies, and this could be due to the late of the patient's presentation.

The clinical course of the disease following the diagnosis was the predominant presentation of single relapse and multiple relapses in one third of the patients with the median course of the disease of 21 months.

This is comparable to previous data that reported a relapse rate of $55 \%$ in the first year [22]. In most of the seropositive patients, relapse occur early with about $55 \%$ of patients having a relapse in the first year and $90 \%$ within the first 5 years [23]. However, among the seropositive patients that we studied $68 \%$ reported a single relapse course during the 3 year follow-up. These patients were on azathioprine treatment since the onset of the disease. This might be the cause of reduction of relapses during the study duration, however further relapses may occur in future. Another explanation could be that relapses in those seropositive patients $(68 \%)$ might have occurred during the disease follow-up duration as a mild relapse in addition to the persistent disability of the patients, which could have passed unnoticed by the patients.

The findings from this study demonstrate that AQP4IgG seropositivity was high with a frequency of $79.3 \%$, samples were analyzed using ELISA, but the cell-based assays are superior to ELISAs. These findings are in agreement with those of international studies [19-21] except a study conducted in Saudi Arabia [21]. In recent years, serum reactivity that target AQP4-IgG is detectable in $60 \%$ to $90 \%$ of patients with NMO. Some studies have indicated that CSF AQP4IgG is present in most patients with positive serum AQP4IgG. AQP4IgG is detectable mostly as a result of passive diffusion from the serum [24]. This could explain the equality of the percentage of CSF and serum AQP4-IgG.

With respect to the treatment and outcome, 26 of the patients $(83.9 \%)$ received treatment in the form of intravenous (IV) methylprednisolone $1 \mathrm{gm} /$ day for 5 days followed by oral prednisolone $60 \mathrm{mg} /$ day tapered down according to the response, and azathioprine $150 \mathrm{mg}$ /day; and a majority $22(71.0 \%)$ of the patients improved with some disability. These results are in accordance to a previous study which stated that $69 \%$ of patients treated with oral prednisolone and azathioprine showed a marked reduction in the relapse rate [25]. That plasma exchange therapy was not available for the majority of patients at that time but now it's available, plasma exchange is very effective in acute relapse of neuromyelitis optica [26]. In Sudan we can deal with the approval of the first expensive NMOSD therapy (Eculizumab) in the future, although most of our patients are of low socioeconomic status.

In our study, there was a significant correlation between the response to treatment and serum Aquaporin4.

\section{Conclusion}

Females were more affected than males and patients older than 40 years were over- represented. The most frequent initial presentation was motor impairment rather than optic neuritis. Neuroimaging demonstrated abnormal MRI of cervical spine in the form of longitudinal hyper-intense lesion involving more than 3 consecutive vertebrae, whilst most patients had a normal MRI of the brain. This study showed a higher seropositivity for AQP4 compared to other countries. Most of seropositive patient's showed a good response to treatment but with residual disabilities.

\section{Abbreviations}

APQ4-lgG: Aquaporin 4 immunoglobulin; CSF: Cerebrospinal fluid; NCNS: National Center for Neurological Sciences; NMOSD: Neuromyelitis optica spectrum diseases; MRI: Magnetic resonance imaging.

\section{Acknowledgements}

The authors would like to acknowledge the National Center for Neurological Science for their support and help.

\section{Authors' contributions}

EIA, the first author extracted the data, analyzed and interpreted it, drafted the manuscript, gave the final approval of the version to be published, agreed to be accountable for all aspects of the work, in addition to that, diagnosed and treated the patients. FG, the second author extracted the data, analyzed and interpreted, drafted the manuscript, gave final approval of the version to be published, and agreed to be accountable for all aspects of the work. AG, the third author, participated in extraction of the data, analyzed and interprets it, drafted the manuscript, gave final approval of the version to be published, and agreed to be accountable for all aspects of the work. All authors read and approved the final manuscript.

\section{Funding}

The study was funded by the authors themselves.

\section{Availability of data and materials}

The datasets used and/or analyzed during the current study are available from the corresponding author upon request.

\section{Ethics approval and consent to participate}

This work was approved by the ethical committee at the National Center for Neurological Science, Khartoum, Sudan reference number 2009-02-09. The ethical approval certificate is attached. All the authors approved the participation of the manuscript. Consent was taken from all participants; a sample of the consent form in Arabic is attached.

\section{Consent for publication}

Not applicable.

Competing interests

The authors declare that they have no competing interests. 


\section{Author details}

${ }^{1}$ Alneelain University, Faculty of Medicine, Khartoum, Sudan. ${ }^{2}$ The National Center for Neurological Sciences, Khartoum, Sudan. ${ }^{3}$ ALMadain College, Khartoum, Sudan.

Received: 3 September 2019 Accepted: 14 February 2020

Published online: 28 February 2020

\section{References}

1. Devic E. Mye'litesubaigue" complique'e de ne'vriteoptique. Bull Med (Paris). 1894:8:1033-4.

2. Wingerchuk DM, Hogancamp WF, O'Brien PC, et al. The clinical course of neuromyelitis optica (Devic's syndrome). Neurology. 1999;53:1107-14.

3. Jung JS, et al. Molecular characterization of an aquaporin CDNA from brain: candidate osmo receptor and regulator of water balance. Proc Natl Acad Sci. 1994;91(26):13052-6.

4. Lucchinetti CF, Mandler RN, McGavern D, et al. A role for humoral mechanisms in the pathogenesis of Devic'sneuromyelitisoptica. Brain. 2002;125:1450-61.

5. Giovannoni G. To test or not to test: NMO-lgG and optic neuritis. Neurology. 2008;70:2192-3.

6. Kim W, Park MS, Lee SH, et al. Characteristic brain magnetic resonance imaging abnormalities in central nervous system aquaporin-4 autoimmunity. Mult Scler. 2010;16:1229-36.

7. Kim W, Kim SH, Huh SY, et al. Brain abnormalities in neuromyelitis optica spectrum disorder. Mult Scler Int. 2012;2012:735486.

8. Matiello M, Lennon VA, Jacob A, et al. NMO-lgG predicts the outcome of recurrent optic neuritis. Neurology. 2008;70:2197-200.

9. Weinshenker BG, Wingerchuk DM, Vukusic S, et al. Neuromyelitis optica IgG predicts relapse after longitudinally extensive transverse myelitis. Ann Neurol. 2006;59:566-9.

10. Claudia FL. The pathology of an autoimmune astrocytopathy: lessons learned from neuromyelitis optica. Brain Pathol. 2014;24(1):83-97.

11. Wingerchuk DM, Lennon VA, Pittock SJ, Lucchinetti CF, Weinshenker BG. Revised diagnostic criteria for neuromyelitis optica. Neurology. 2006;66(10):1485-9.

12. Ibrahim EAA, Gassoum A. The patterns of clinical presentation of multiple sclerosis in patients admitted to the National Center of Neurological Sciences, Khartoum, Sudan 2018. Mult Scler Relat Disord. 2018;26:238.
13. Asgari N, Lillevang ST, Skejoe HP. A population-based study of neuromyelitis optica in Caucasians. Neurology. 2011;76:1589-95.

14. Cabre P. Environmental changes and epidemiology of multiple sclerosis in the French West Indies. J Neurol Sci. 2009;286:58-61.

15. Aboul-Enein F, Seifert-Held T, Mader S. Neuromyelitisoptica in Austria in 2011: to bridge the gap between neuroepidemiological research and practice in a study population of 8.4 million people. PLoS ONE. 2013;8:e79649.

16. Cabrera-Gomez JA, Kurtzke JF, Gonzalez-Quevedo A. An epidemiological study of neuromyelitis optica in Cuba. J Neurol. 2009;256:35-44.

17. Cossburn M, Tackley G, Baker K. The prevalence of neuromyelitis optica in South East Wales. Eur J Neurol. 2011:19:655-9.

18. Long Y, Qiu W, Lu Z, et al. Aquaporin 4 antibodies in the cerebrospinal fluid is helpful in diagnosing Chinese patients with neuromyelitis optica. Neuroimmunomodulation. 2012;19:96-102.

19. Kira J. Neuromyelitis optica and opticospinal multiple sclerosis: mechanisms and pathogenesis. Pathophysiology. 2011;18:69-79.

20. Pandit L. Neuromyelitis optica antibody (NMO lgG) status in Indian patients with multiple sclerosis and allied demyelinating disorders. Neurol Asia. 2008;13:175-8.

21. Al-Khathaami AM, Yunus F, Alamgir MJ, Kojan S, Aljumah M. Neuromyelitis optica and neuromyelitis optica-lgGseropositivity in Saudis with demyelinating diseases of the central nervous system. Neurol Asia. 2014;19(3):295-300.

22. Wingerchuk DM. Diagnosis and treatment of neuromyelitis optica. Neurologist. 2007;13(1):2-11.

23. Wingerchuk DM. Neuromyelitisoptica. The Internatl MS Jou. 2006;13(2):42-50.

24. Takahashi T, et al. Anti AQP4 Anti Ab is involved in the pathogeneses of NMO; study on antibody titre. Brain. 2007;130:1235-43.

25. Mandler RN, Ahmed W, Dencoff JE. Neuromyelitis optica: study of treatment with prednisone and azathioprine. Neurology. 2008;51:1219-20.

26. Jasiak-Zatonska M, Kalinowska-Lyszczarz A, Michalak S, Kozubski W. The immunology of neuromyelitis optica-current knowledge, clinical implications, controversies and future perspectives. Int J Mol Sci. 2016;17:273.

\section{Publisher's Note}

Springer Nature remains neutral with regard to jurisdictional claims in published maps and institutional affiliations.
Ready to submit your research? Choose BMC and benefit from:

- fast, convenient online submission

- thorough peer review by experienced researchers in your field

- rapid publication on acceptance

- support for research data, including large and complex data types

- gold Open Access which fosters wider collaboration and increased citations

- maximum visibility for your research: over 100M website views per year

At BMC, research is always in progress.

Learn more biomedcentral.com/submissions 